\title{
Rigidity Dependence of the Long-Term Variations of Galactic Cosmic-Ray Intensity in Relation to the Interplanetary Magnetic-Field Turbulence: 1968-2002
}

\author{
M. Siluszyk $\cdot$ K. Iskra $\cdot$ M.V. Alania
}

Received: 10 December 2013 / Accepted: 8 July 2014 / Published online: 23 July 2014

(C) The Author(s) 2014. This article is published with open access at Springerlink.com

\begin{abstract}
We studied the relationship between the power-law exponent $\gamma$ on the rigidity $R$ of the spectrum of galactic cosmic-ray (GCR) intensity variation $\left(\delta D(R) / D(R) \propto R^{-\gamma}\right)$ and the exponents $v_{y}$ and $v_{z}$ of the power spectral density (PSD) of the $B_{y}$ and $B_{z}$ components of the interplanetary magnetic field (IMF) turbulence (PSD $\sim f^{-v}$, where $f$ is the frequency). We used the data from neutron monitors and IMF for the period of 1968-2002. The exponents $v_{y}$ and $v_{z}$ were calculated in the frequency interval $\Delta f=f_{2}-f_{1}=3 \times 10^{-6} \mathrm{~Hz}$ of the resonant frequencies $\left(f_{1}=1 \times 10^{-6} \mathrm{~Hz}, f_{2}=4 \times 10^{-6} \mathrm{~Hz}\right)$ that are responsible for the scattering of GCR particles with the rigidity range detected by neutron monitors. We found clear inverse correlations between $\gamma$ and $v_{y}$ or $v_{z}$ when the time variations of the resonant frequencies were derived from in situ measurements of the solar wind velocity $U_{\text {sw }}$ and IMF strength $B$ during 1968-2002. We argue that these inverse relations are a fundamental feature in the GCR modulation that is not restricted to the analyzed years of 1968-2002.
\end{abstract}

Keywords Galactic cosmic ray intensity · Interplanetary magnetic field turbulence $\cdot$ Long period variations $\cdot$ Rigidity dependence

\section{Introduction}

The relationship between the power-law exponent $\gamma$ on the rigidity $R$ of the spectrum of galactic cosmic-ray (GCR) intensity variations $\left(\delta D(R) / D(R) \propto R^{-\gamma}\right)$ and the exponent $v$

\author{
M. Siluszyk (凶) · K. Iskra · M.V. Alania \\ Institute of Mathematics and Physics, Siedlce University, Siedlce, Poland \\ e-mail: marek.siluszyk@uph.edu.pl \\ K. Iskra \\ e-mail: krzysztof.iskra@uph.edu.pl \\ M.V. Alania \\ e-mail: michael.alania@uph.edu.pl
}

M.V. Alania

Institute of Geophysics, Tbilisi State University, Tbilisi, Georgia 
of the power spectral density (PSD) of the interplanetary magnetic-field (IMF) turbulence (PSD $\sim f^{-v}$, where $f$ is the frequency) and its long-term changes are one of the most interesting research topics on the GCR modulation in the heliosphere. Several fundamental processes such as diffusion, convection, changes in GCR particle energy due to interactions with the radial expansion of the solar wind, and drifts due to curvature and gradient of the regular IMF and on the heliospheric current sheet (HCS) are responsible for the GCR intensity variations in the heliosphere.

The roles of various processes in the GCR intensity modulation depend upon the rigidity of GCR particles and the spatial and time scales of GCR intensity variations. It is shown that, in the first approximation, about 75-80\% of the 11-year variation of the GCR intensity can be interpreted based on the anisotropic diffusion-convection model of GCR propagation (Dorman, 2001; Alania, 2002; Alania, Iskra, and Siluszyk, 2010; Strauss and Potgieter, 2014) for particles of rigidity range 5-35 GV that are detected by neutron monitors (NMs). According to the quasi-linear theory (QLT) by Jokipii (1971) and Shalchi (2009), there should be a relationship between the parallel diffusion coefficient $K_{/ /}$ and rigidity $R$ of GCR particles, as $K_{/ /} \propto R^{\alpha}$ for the rigidity range of NMs. The parameter $\alpha$ is represented as $\alpha=2-v$, where $v$ is the exponent of the PSD of the IMF turbulence.

Comprehensive studies based on numerical solutions of Parker's transport equation and neutron monitor data (Alania and Iskra, 1995; Alania, 2002; Alania, Iskra, and Siluszyk, 2003) have shown that the exponent $\gamma$ is proportional to $\alpha$. We have also shown that the exponent $\gamma$ demonstrates clear 11-year variations (Alania, 2002; Alania, Iskra, and Siluszyk, 2003; Siluszyk, Wawrzynczak, and Alania, 2011).

Figure 1 presents temporal variations in $\gamma$ (middle panel) and sunspot number (SSN, bottom panel). The top panel shows the GCR intensity variations $J$ from Moscow neutron monitor data rescaled to free space using the coupling coefficients by Yasue et al. (1982) and Alania, Iskra, and Siluszyk (2010). In the entire period of 1968-2002, there exists a clear negative correlation between $J$ and $\gamma$ (correlation coefficient $r=-0.62 \pm 0.12$ ) and a positive correlation between SSN and $\gamma(r=0.70 \pm 0.11)$, in spite of some time delays between the peaks and valleys in $J, \mathrm{SSN}$, and $\gamma$ of individual 11-year cycles.

The scattering of GCR particles in the heliosphere is generally caused by the turbulence of the $B_{y}$ and $B_{z}$ components of the IMF (Jokipii, 1971; Bieber, Mathaeus, and Smith, 1994), although the roles of the two components are not equal at all. The average power of the PSD of the $B_{y}$ component $\left(\sim f v_{y}\right)$ is more than twice as large as the average power of the $B_{z}$ component $\left(\sim f v_{z}\right)$. Nevertheless, there is a good correlation between the temporal variations in the exponents $v_{y}$ and $v_{z}$ (Alania, Iskra, and Siluszyk, 2009).

Therefore, in spite of the important role in the GCR modulation ascribed to the $B_{y}$ component of the IMF (Alania, Iskra, and Siluszyk, 2009), we here consider the behaviors of both exponents $v_{y}$ and $v_{z}$ versus solar activity. Our finding $\gamma \propto 2-v_{y}$ (Alania and Iskra, 1995; Alania, 2002; Alania, Iskra, and Siluszyk, 2003) is consistent with the inverse correlation between $\gamma$ and $v_{y}$, as expected from the QLT by Jokipii (1971) and Shalchi (2009).

We have calculated the annual average values of $v_{y}$ and $v_{z}$ (Alania, Iskra, and Siluszyk 2008a, 2008b, 2009, 2010) for the period 1968-2002 and divided them into three subperiods - I: 1968 - 1976, II: 1977 - 1989, and III: $1990-2002$. Period I corresponds to the second half of solar cycle 20. Period II consists of cycle 21 and the first half of cycle 22 . Period III contains the second half of cycle 22 and the first half of cycle 23 . We have found high inverse correlations between $\gamma$ and $v_{y}$ or $v_{z}$ for period II; while the relationships between $\gamma$ and $v_{y}$ or $v_{z}$ are less obvious for periods I and III.

The aim of this paper is i) to study the relationship between the power-law exponent $\gamma$ of the long-term variations in the GCR intensity $\left(\delta D(R) / D(R) \propto R^{-\gamma}\right)$ and the exponents 

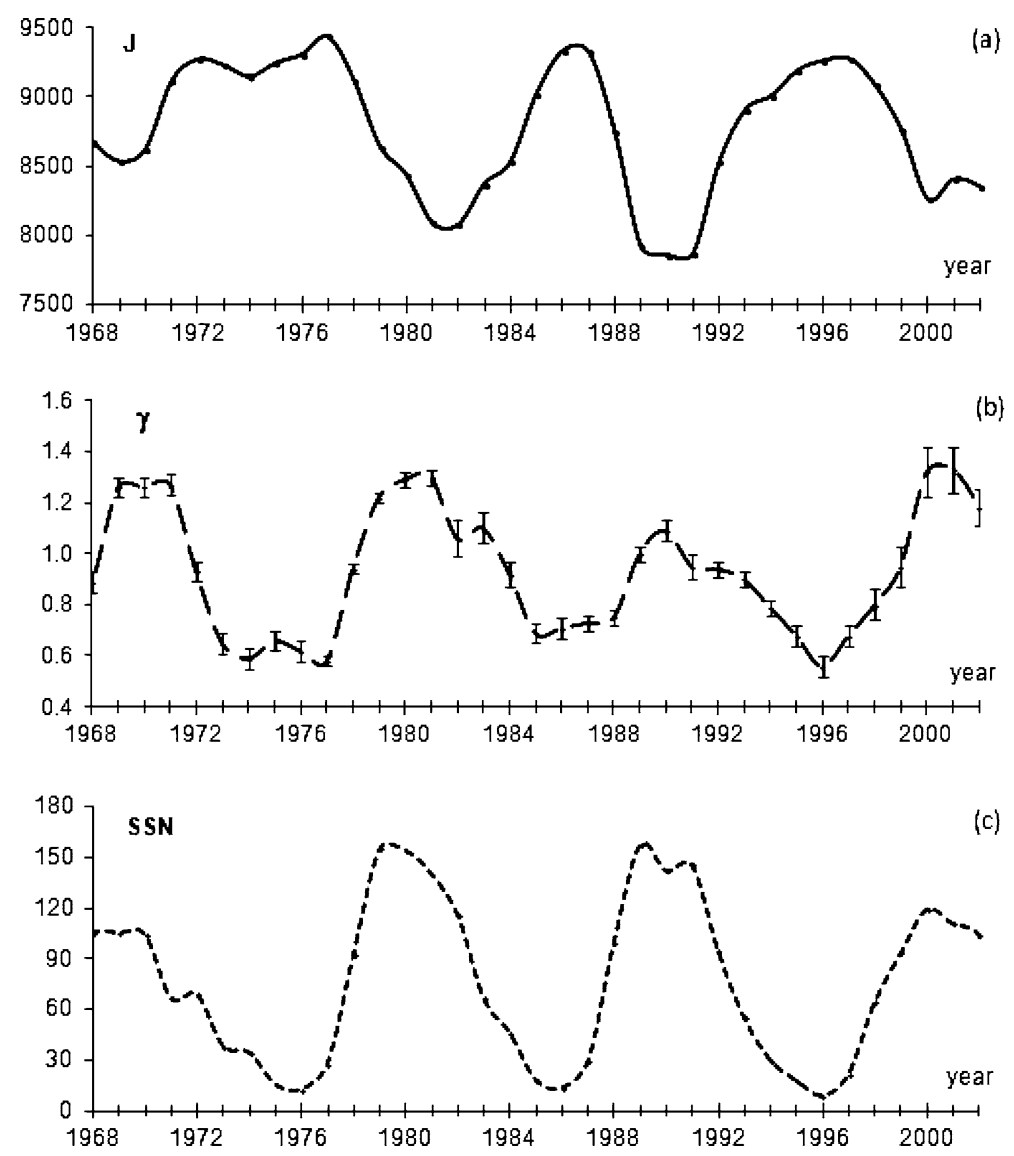

Figure 1 Temporal variations in the annual means for the period of 1968-2002 of (a) the GCR intensity measured by the Moscow neutron monitor, (b) the rigidity spectrum exponent $\gamma$ of the GCR intensity variations, and (c) the sunspot number.

$v_{y}$ and $v_{z}$ of the PSD of the $B_{y}$ and $B_{z}$ components of the IMF turbulence, and ii) to show that the inverse correlations between $\gamma$ and $v_{y}$ or $v_{z}$ are a fundamental feature in the GCR modulation not restricted only to the analyzed period, as expected from the QLT of GCR particles for the rigidity range of NMs.

\section{Observational Data and Analysis Methods}

We have used data of NMs and IMF in 1968-2002. Table 1 presents the list of NMs used to calculate the exponent $\gamma$ (see Appendix A). Unfortunately, we need a sufficient number of NMs that functioned well for a long period to calculate $\gamma$. To provide at least an acceptable accuracy for the calculated value of $\gamma$ we used carefully selected appropriate NMs for each chosen period of solar activity.

Alania, Iskra, and Siluszyk $(2009,2010)$ calculated the annual average values of $v_{y}$ and $v_{z}$ by the method given in Appendix B. This method (which we call the first method) assumes the same frequency interval $\Delta f=f_{2}-f_{1}=3 \times 10^{-6} \mathrm{~Hz}$ and constant resonant frequencies 
Table 1 Neutron monitors used to calculate $\gamma$, denoted by ' + ' in the analysis periods.

\begin{tabular}{|c|c|c|c|c|c|c|c|c|c|c|}
\hline \multicolumn{3}{|c|}{ Reference point (RP): } & \multicolumn{2}{|c|}{1965} & \multicolumn{2}{|c|}{1976} & \multicolumn{2}{|c|}{1986} & \multicolumn{2}{|c|}{1996} \\
\hline No. & Stations & $\begin{array}{l}\text { Cut-off } \\
\text { rigidity } \\
{[\mathrm{GV}]}\end{array}$ & 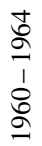 & 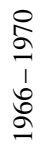 & 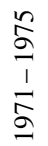 & 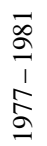 & $\begin{array}{l}\mathscr{2} \\
\stackrel{2}{\sigma} \\
\stackrel{1}{\alpha} \\
\stackrel{\infty}{\varrho}\end{array}$ & 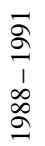 & 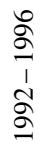 & 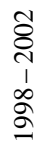 \\
\hline 1 & Apatity & 0.65 & - & - & - & - & - & - & - & + \\
\hline 2 & Climax & 3.03 & + & + & + & + & + & + & + & + \\
\hline 3 & Deep River & 1.02 & + & - & - & + & + & + & - & - \\
\hline 4 & Goose Bay & 0.52 & - & - & - & + & + & + & + & - \\
\hline 5 & Haleakala-Huancayo & 13.4 & + & + & + & + & + & + & + & + \\
\hline 6 & Hermanus & 4.90 & - & + & - & + & + & + & - & + \\
\hline 7 & Inuvik & 0.18 & - & + & + & + & + & + & + & - \\
\hline 8 & Jungfraujoch & 4.48 & - & - & - & + & - & - & - & - \\
\hline 9 & Kergulen Is & 1.19 & - & - & + & - & - & - & - & - \\
\hline 10 & Kiel & 2.29 & + & + & + & + & + & + & + & + \\
\hline 11 & Mc Murdo & 0.01 & - & - & - & - & - & - & - & + \\
\hline 12 & Moscow & 2.46 & + & + & + & + & + & + & + & + \\
\hline 13 & Mt Norikura & 11.39 & - & - & - & - & + & - & - & - \\
\hline 14 & Mt Washington & 1.24 & - & + & + & - & + & - & + & - \\
\hline 15 & Pic-du-Midi & 5.36 & - & + & + & - & - & - & - & - \\
\hline 16 & Potchestroom & 7.30 & - & - & - & + & + & + & + & + \\
\hline 17 & Rome & 6.32 & - & - & - & - & - & - & - & + \\
\hline 18 & Tbilisi & 6.91 & - & - & - & + & + & + & - & - \\
\hline
\end{tabular}

( $f_{1}=1 \times 10^{-6} \mathrm{~Hz}, f_{2}=4 \times 10^{-6} \mathrm{~Hz}$ ), corresponding to the constant solar wind velocity $U_{\text {sw }}=432.4 \mathrm{~km} \mathrm{~s}^{-1}$ and IMF strength $B=4.6 \mathrm{nT}$ for the whole period $1968-2002$.

On the other hand, the solar wind velocity $U_{\text {sw }}$ and the IMF strength $B$ might have varied significantly from year to year during $1968-2002$ and the frequencies $f_{1}$ and $f_{2}$ might have changed correspondingly. Therefore, it is interesting to see how the correlations between $\gamma$ and $v_{y}$ or $v_{z}$ change when $v_{y}$ and $v_{z}$ are calculated for the frequency interval $\Delta f=f_{2}-f_{1}$ obtained based on the solar wind velocity $U_{\text {sw }}$ and the IMF strength $B$ that change from year to year (hereafter called the second method).

Figures $2 \mathrm{a}$ and $2 \mathrm{~b}$ show the variations in the solar wind velocity $U_{\mathrm{sw}}$ and the IMF strength $B$ in the period $1968-2002$. The IMF strength $B$ (Figure 2a) shows a quasi-11-year periodicity, while the solar wind velocity $U_{\text {sw }}$ (Figure 2 b) does not show a clear 11-year periodicity.

To calculate the resonant frequency $f_{\text {res }}$ and the corresponding frequency interval $\Delta f$ from the values of $U_{\text {sw }}$ and $B$ that vary with the solar activity, we used the formula $f_{\text {res }}=$ $\frac{300}{2 \pi} \frac{U_{\mathrm{sw}} \cdot B}{R}$ by Jokipii and Coleman (1968) and Jokipii (1971). The temporal variations in $f_{\text {res }}$ thus derived for GCR particles of rigidity $R=10 \mathrm{GV}$ are presented in Figure 3.

By comparing Figures $2 \mathrm{a}, 2 \mathrm{~b}$, and 3 , one can see that the time profile of $f_{\text {res }}$ is mostly caused by the variations in $B$ from year to year that clearly show quasi-11-year periodicity (correlation coefficient $r$ between $f_{\text {res }}$ and $B$ is $r=0.95 \pm 0.01$ ), while the role of the solar wind velocity $U_{\text {sw }}$ is not significant (correlation coefficient $r$ between $f_{\text {res }}$ and $U_{\text {sw }}$ is only $r=0.31 \pm 0.03$ ). As an example, Figure 4 presents variations in $f_{\text {res }}$ versus rigidity $R$ for 



Figure 2 Temporal variations in (a) the solar wind velocity $U_{\mathrm{sw}}$ and (b) the IMF strength $B$ during the period of $1968-2002$.

given $B$ and $U_{\mathrm{sw}}$ in the minimum $\left(1986, B=3.7 \mathrm{nT}, U_{\mathrm{sw}}=441.6 \mathrm{~km} \mathrm{~s}^{-1}\right)$ and maximum (1989, $\left.B=5.8 \mathrm{nT}, U_{\mathrm{sw}}=435.5 \mathrm{~km} \mathrm{~s}^{-1}\right)$ epochs of solar activity.

The ranges of resonant frequencies and the corresponding intervals $\Delta f=f_{1}-f_{2}=$ $(3-4) \times 10^{-6} \mathrm{~Hz}$ for which the exponents $v_{y}$ and $v_{z}$ are calculated for variable $U_{\mathrm{sw}}$ and $B$ are presented in Table 2. For each chosen period the values of $f_{\text {res }}$ are different, but the frequency intervals $\Delta f$ remain nearly constant.

\section{Results and Discussion}

By using the resonant frequencies $f_{\text {res }}$ and frequency intervals $\Delta f$ given in Table 2, we have calculated the corresponding annual values of the exponents $v_{y}$ and $v_{z}$. Figure 5a presents temporal variations in the exponents $v_{y}$ derived from the first method (dotted line) and the second method (dashed line) in periods I, II, and III, together with the variations in $\gamma$ (solid line). Figure $5 \mathrm{~b}$ presents the results for $v_{z}$.

To carry out a more detailed study on the relations between $\gamma$ and $v_{y}$ or $v_{z}$, we evaluated the correlation coefficients $r_{1}\left(\gamma ; v_{y}\right)$ and $r_{1}\left(\gamma ; v_{z}\right)$ when the values $v_{y}$ and $v_{z}$ were derived 




Figure 3 Annual variations in the resonant frequency $f_{\text {res }}$ for GCR particles of rigidity $R=10$ GV from 1968 to 2002 .

Figure 4 Temporal variations in the resonant frequency $f_{\text {res }}$ versus rigidity $R$ for given $B$ and $U_{\text {sw }}$ for the minimum epoch 1986 (the dotted line) and the maximum epoch 1989 (the solid line) of solar activity.



from the first method, and $r_{2}\left(\gamma ; v_{y}\right)$ and $r_{2}\left(\gamma ; v_{z}\right)$ derived from the second method for three periods I, II, and III separately, and for the whole period 1968-2002 (Table 3).

A high inverse correlation is observed in period II for both $v_{y}$ and $v_{z}$, regardless of whether we used the first or the second method. This conclusion is valid even if the values of $v_{y}$ and $v_{z}$ were derived from different frequency ranges of the IMF turbulence shown in Table 2 over cycle 21 (1976-1986).

A distinction in correlations with $\gamma$ for $v_{y}$ and $v_{z}$ is observed for periods I and III. A clear inverse correlation takes place between $\gamma$ and $v_{y}$ or $v_{z}$ when the second method is used, namely, when the changes in the resonant frequency that depend on the solar activity level 
Table 2 Frequency ranges and frequency intervals $\Delta f$ calculated for different time intervals of $1968-2002$.

\begin{tabular}{lll}
\hline Periods & Frequency range $[\mathrm{Hz}]$ & $\Delta f[\mathrm{~Hz}]$ \\
\hline 1968 & $7.9 \times 10^{-7}-3.8 \times 10^{-6}$ & 3.0 \\
$1969-1970$ & $2.1 \times 10^{-6}-5.8 \times 10^{-6}$ & 3.7 \\
$1971-1975$ & $7.9 \times 10^{-7}-3.8 \times 10^{-6}$ & 3.0 \\
$1976-1977$ & $7.6 \times 10^{-7}-3.8 \times 10^{-6}$ & 3.0 \\
$1978-1980$ & $1.7 \times 10^{-6}-5.8 \times 10^{-6}$ & 4.1 \\
$1981-1982$ & $1.0 \times 10^{-6}-4.0 \times 10^{-6}$ & 3.0 \\
1983 & $1.7 \times 10^{-6}-5.8 \times 10^{-6}$ & 4.1 \\
$1984-1987$ & $7.6 \times 10^{-7}-3.8 \times 10^{-6}$ & 3.0 \\
1988 & $1.0 \times 10^{-6}-4.0 \times 10^{-6}$ & 3.0 \\
$1989-1992$ & $1.7 \times 10^{-6}-5.8 \times 10^{-6}$ & 4.1 \\
$1993-1998$ & $7.0 \times 10^{-7}-3.7 \times 10^{-6}$ & 3.0 \\
$1999-2000$ & $1.7 \times 10^{-6}-5.2 \times 10^{-6}$ & 3.5 \\
2001 & $1.0 \times 10^{-6}-4.0 \times 10^{-6}$ & 3.0 \\
2002 & $1.7 \times 10^{-6}-5.2 \times 10^{-6}$ & 3.5 \\
\hline
\end{tabular}

are taken into account. It is likely that odd and even 11-year solar cycles may show different turbulence structures of IMF. We do not exclude, for example, the rearrangements of the IMF turbulence depending on the sequence of the reversal of the Sun's global magnetic field; for solar cycles 20 and 22 the Sun's global field changed from negative $(A<0)$ to positive $(A>0)$ polarities, while solar cycle 21 was in the opposite situation.

The inverse correlation between $\gamma$ and $v$ in periods I and III (during cycles 20 and 22) that was found by allowing the resonant frequencies to vary with the solar activity level should be considered as normal because it agrees with the theory of scattering of GCR particles in the interplanetary space. On the other hand, the high inverse correlations between $\gamma$ and $v_{y}$ or $v_{z}$ regardless of the changes in the IMF strength could be exceptional or accidental.

Figure 6a shows the temporal variations in $\gamma$ (solid line) and $v_{y}$ derived from the first (dashed line) and second method (dotted line) for the whole period 1968-2002. Figure 6b shows the same results for the exponent $v_{z}$.

Figure 6 shows that the exponent $\gamma$ has clear 11-year periodicity with its maxima at the maximum epochs of solar activity, while the maxima of $v_{y}$ and $v_{z}$ occur at the minimum epochs. These results indicate a significant rearrangement of the IMF turbulence structure from the maximum to the minimum epochs of solar activity, although its detailed properties may vary among individual 11-year cycles.

We found that the variations in $v_{y}$ and $v_{z}$ according to the level of solar activity play a central role in determining the exponent $\gamma$, or more generally, in characterizing long-term variations in the GCR intensity. Thus, one can conclude that the inverse relations between $\gamma$ and $v$ are a fundamental feature in the GCR modulation, not only restricted to the analyzed years of $1968-2002$, but found generally in any period.

\section{Summary}

1. Temporal variations in the exponents $v_{y}$ and $v_{z}$ of the PSD of the IMF obtained by in situ measurements of the $B_{y}$ and $B_{z}$ components in near-Earth space reflect the average structure of the IMF turbulence in the vicinity of the heliosphere where the long-term 

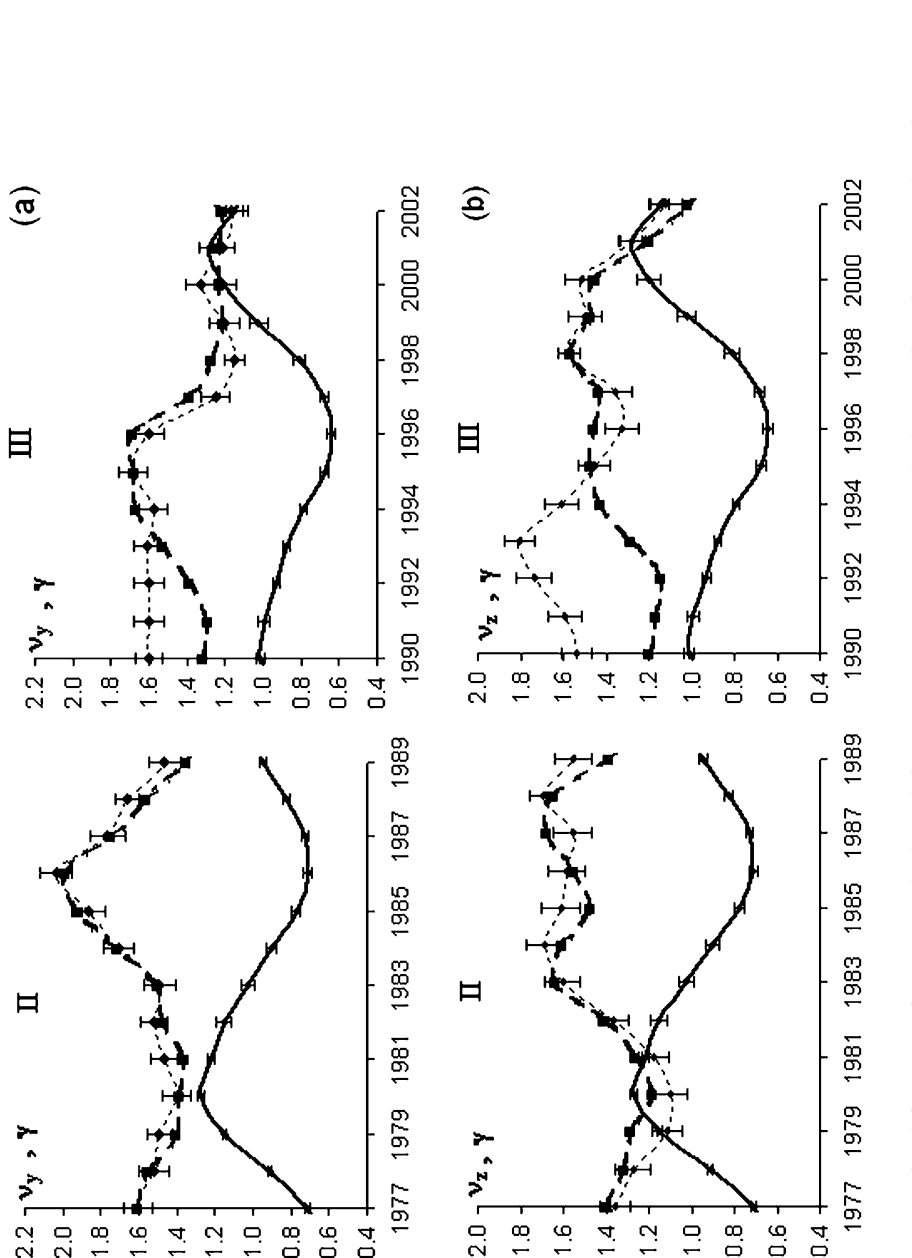

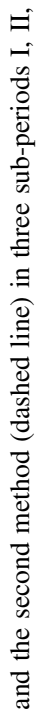

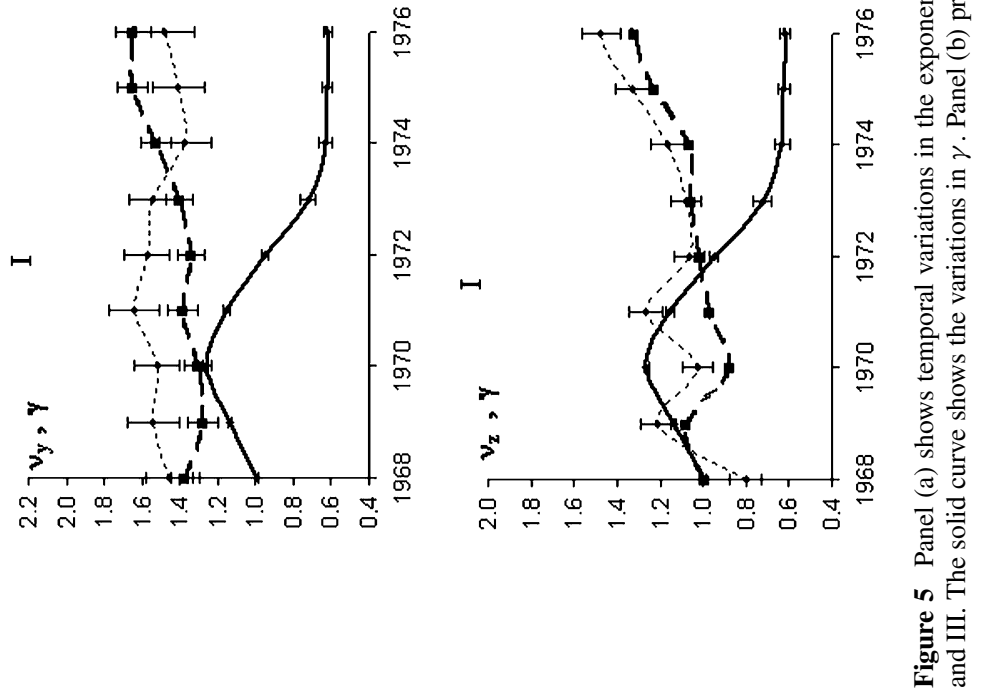


Table 3 Correlation coefficients $r$ between $\gamma$ and $v_{y}$ or $v_{z}$. The first and the second methods were used to derive $v_{y}$ or $v_{z}$ in $r_{1}$ and $r_{2}$, respectively.

\begin{tabular}{llllll}
\hline & Period & $r_{1}\left(\gamma ; v_{\mathrm{y}}\right)$ & $r_{2}\left(\gamma ; v_{\mathrm{y}}\right)$ & $r_{1}\left(\gamma ; v_{z}\right)$ & $r_{2}\left(\gamma ; v_{z}\right)$ \\
\hline I & $1968-1976$ & $0.64 \pm 0.17$ & $-0.86 \pm 0.11$ & $-0.43 \pm 0.20$ & $-0.78 \pm 0.14$ \\
II & $1977-1989$ & $-0.78 \pm 0.11$ & $-0.78 \pm 0.11$ & $-0.69 \pm 0.13$ & $-0.66 \pm 0.13$ \\
III & $1990-2002$ & $-0.43 \pm 0.16$ & $-0.78 \pm 0.12$ & $-0.19 \pm 0.18$ & $-0.54 \pm 0.15$ \\
& $1968-2002$ & $-0.32 \pm 0.17$ & $-0.66 \pm 0.13$ & $-0.32 \pm 0.17$ & $-0.38 \pm 0.16$ \\
\hline
\end{tabular}
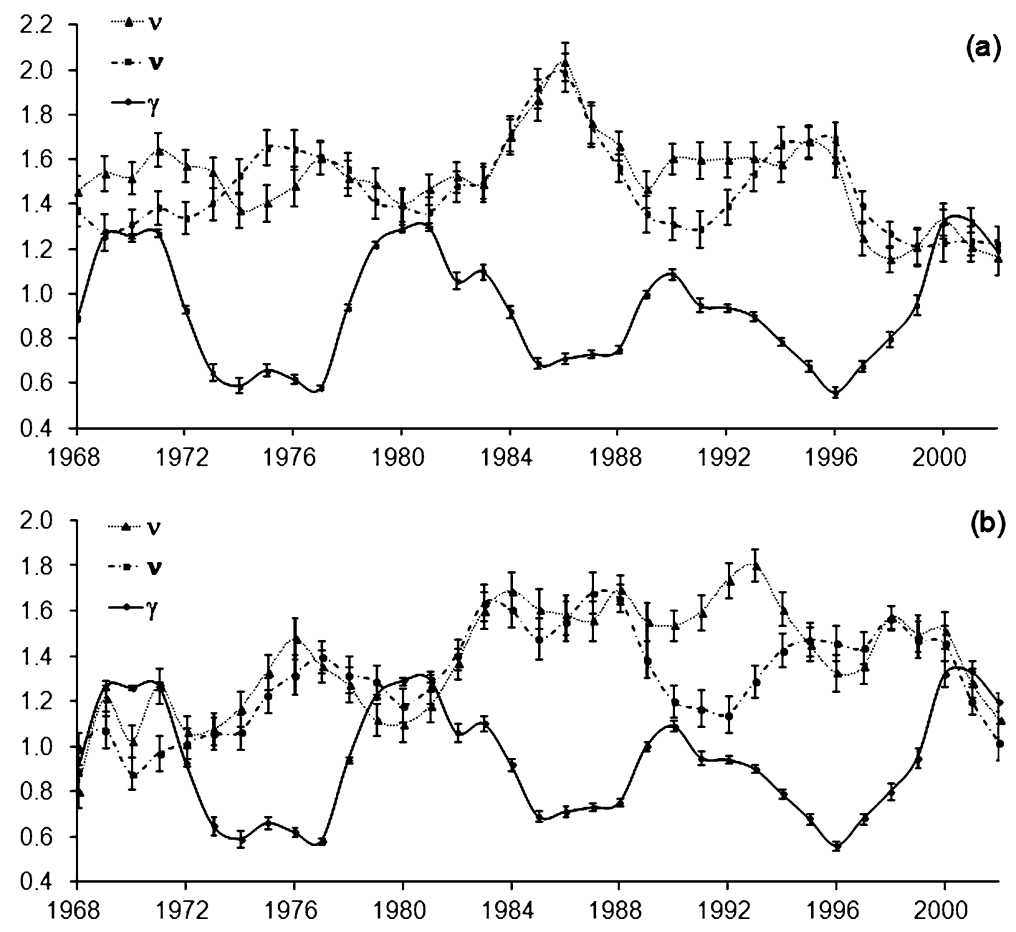

Figure 6 Panel (a) shows the temporal variations in $\gamma$ (solid line) and $v_{y}$ derived from the first (dotted line) and second method (dashed line) for the whole period 1968-2002. Panel (b) shows the results for the exponent $v_{z}$.

variations of the GCR modulation occur. Under this assumption, both $v_{y}$ and $v_{z}$, which clearly show 11-year periodicity, can be considered as the central parameters controlling the modulation of GCR in the rigidity range of neutron monitor measurements.

2. We showed that for GCR particles of rigidity $10 \mathrm{GV}$, the resonant frequencies are lower in the minimum epochs than in the maximum epochs of solar activity for 1968-2002. These changes in the resonant frequencies of the IMF turbulence are primarily due to the quasi-11-year periodicity in the strength $B$ of the IMF.

3. A significant high inverse correlation is observed between $\gamma$ and $v_{y}$ or $v_{z}$ in period II (1977 - 1989 which includes solar cycle 21). This correlation persists even if $v_{y}$ and $v_{z}$ are calculated for constant values of $B$ and $U_{\text {sw }}$ corresponding to resonant frequencies averaged over the whole period of $1968-2002$, or if the resonant frequencies are varied 
according to the level of solar activity in period II. The values of $v_{y}$ and $v_{z}$ do not change if the frequency interval $\Delta f=(3-4) \times 10^{-6} \mathrm{~Hz}$ is shifted in frequency as a function of time during cycle 21 . This is possibly due to a unique condition for solar cycle 21 , which is characterized by nearly homogeneous and isotropic turbulence of the IMF in space.

4. On the other hand, for periods I and III (solar cycles 20 and 22) a clear inverse correlation between $\gamma$ and $v_{y}$ or $v_{z}$ only occurs if the resonant frequencies of turbulence are varied according to the level of solar activity year by year.

5. We argue that the inverse correlation between $\gamma$ and $v$ during cycles 20 and 22, which is found by changing the resonant frequencies with the level of solar activity (in our case determined by the values of IMF strength), should be considered as a normal situation, consistent with the theory of scattering of GCR particles in interplanetary space. On the other hand, the case of solar cycle 21, in which the inverse correlation between $\gamma$ and $v$ is high and does not depend on the changes in IMF strength with solar activity, should be considered as an exceptional case.

Acknowledgements We thank the referee for the helpful comments and suggestions. We are very grateful to the providers of solar, interplanetary, neutron monitor, and geomagnetic data used in this study. The authors thank the people who created and maintain the websites http://spidr.ngdc.noaa.gov/spidr/, http://omniweb. gsfc.nasa.gov/index.html, http://cr0.izmiran.rssi.ru/common/links.htm, and http://www.nmdb.eu.

Open Access This article is distributed under the terms of the Creative Commons Attribution License which permits any use, distribution, and reproduction in any medium, provided the original author(s) and the source are credited.

\section{Appendix A: Calculation of Exponent $\gamma$}

Neutron monitor data were used to calculate the exponent $\gamma$ on the rigidity $R$ of the spectrum of GCR intensity variations $\left(\frac{\delta D(R)}{D(R)}=A R^{-\gamma}\right)$ in the period of 1960-2002 (Siluszyk et al., 2005; Alania, Iskra, and Siluszyk, 2008a, 2008b, 2009, 2010). These were based on Alania, Iskra, and Siluszyk (2003), who calculated the exponent $\gamma$ using thoroughly selected monthly average data of neutron monitors in the period of 1968-2002, including four ascending and four descending phases of solar activity in the $A>0$ and the $A<0$ epochs. The criterion for data selection adopted in these studies was the continuous operation of neutron monitors with different cut-off rigidities throughout the analyzed period. The magnitude $J_{i}^{k}$ of monthly averaged GCR intensity variations from the $i$ th neutron monitor was calculated as $J_{i}^{k}=\frac{N_{k}-N_{0}}{N_{0}}$, where $N_{k}$ is the running monthly average count rate (months $k=1,2,3, \ldots, 12)$ and $N_{0}$ is the monthly average count rate for the year of highest intensity (usually in the minimum epoch of solar activity). The count rate of the highest intensity is accepted as the $100 \%$ level. The list of selected neutron monitors is presented in Table 1. The magnitude $J_{i}^{k}$ of monthly averaged GCR intensity variations measured by the $i$ th neutron monitor with the geomagnetic cut-off rigidity $R_{i}$ and the average atmospheric depth $h_{i}$ is defined by Dorman (1975) as

$$
J_{i}^{k}=\int_{R_{i}}^{R_{\max }}\left(\frac{\delta D(R)}{D(R)}\right)_{k} W_{i}\left(R, h_{i}\right) \mathrm{d} R,
$$

where $(\delta D(R) / D(R))_{k}$ is the rigidity spectrum of the GCR intensity variations for the $k$ th month; $W_{i}\left(R, h_{i}\right)$ is the coupling coefficient for the neutron component of GCR by Dorman (1975) and Yasue et al. (1982); $R_{\max }$ is the upper limit in rigidity beyond which the GCR 
intensity variation vanishes. For a power-law rigidity spectrum $(\delta D(R) / D(R))_{k}=A R^{-\gamma_{k}}$ one can write

$$
J_{i}^{k}=A_{i}^{k} \int_{R_{i}}^{R_{\max }} R^{-\gamma_{k}} W_{i}\left(R, h_{i}\right) \mathrm{d} R,
$$

where $A_{i}^{k}$ is the magnitude of the GCR intensity variations rescaled to the heliosphere (free space). From Equation (2) we obtain

$$
A_{i}^{k}=J_{i}^{k} / \int_{R_{i}}^{R_{\max }} R^{-\gamma_{k}} W_{i}\left(R, h_{i}\right) \mathrm{d} R .
$$

The values of $A_{i}^{k}$ should be the same (within the accuracy of the calculations) for any $i$ th neutron monitor if the values of parameters $\gamma_{k}$ and $R_{\max }$ are properly determined. A similarity of the values of $A_{i}^{k}$ for various neutron monitors is an essential argument to affirm that the data from a particular neutron monitor and the method of calculations for $\gamma_{k}$ are reliable. To find the temporal variations in the exponent $\gamma_{k}$ (months $k=1,2,3, \ldots, 12$ ), a minimization of the expression

$$
\varphi=\sum_{i}^{n}\left(A_{i}^{k}-A^{k}\right)^{2}
$$

(where $A^{k}=\frac{1}{n} \sum_{i}^{n} A_{i}^{k}$ and $n$ is the number of neutron monitors) was provided by Siluszyk et al. (2005) and Alania, Iskra, and Siluszyk (2008a, 2008b, 2009, 2010). The values of the expression $\int_{R_{i}}^{R_{\max }} R^{-\gamma_{k}} W_{i}\left(R, h_{i}\right) \mathrm{d} R$ for ranges of $R_{\max }$ (from $30 \mathrm{GV}$ up to $200 \mathrm{GV}$ with a step of $10 \mathrm{GV}$ ) and $\gamma$ (from 0 to 2 with a step of 0.05 ) were calculated based on the method presented in Dorman (1975) and Yasue et al. (1982). The upper limit in rigidity, $R_{\max }$, is taken to be $100 \mathrm{GV}$. This assumption was regarded as reasonable for the 11-year variation of the GCR intensity by Gushchina et al. (2008). The minimization of $\varphi$ for the smoothed monthly means (with the interval of 13 months) of the GCR intensity variations was carried out with respect to $\gamma_{k}$ for the neutron monitors given in Table 1.

\section{Appendix B: Calculation of Exponent v}

We collected the data of the $B_{y}$ component of the IMF for the period of 1968-2002 from http://spidr.ngdc.noaa.gov. Then we calculated the power spectrum density (PSD) and its exponent $v$ on the frequency $f$ by the method of Blackman and Tukey by Lyons (1996). First of all, we calculated the autocorrelation function; if $\left\{B_{y_{i}}\right\}(i=1, \ldots, N)$ is a series of daily values of $B_{y}$ of the IMF, the autocorrelation function $R_{r}(r=0,1, \ldots, m)$ is given as

$$
R_{r}=\frac{1}{N-r} \sum_{i=1}^{N-r} B_{y_{i}} B_{y_{i+r}}
$$

where $N=365$ and $m=182$. We then calculated the discrete Fourier transform of the autocorrelation function, $\operatorname{PSD}_{k},(k=0,1, \ldots, m)$ from the formula

$$
\operatorname{PSD}_{k}=2 \Delta t\left(R_{0}+2 \sum_{r=1}^{m-1} R_{r} \cos \frac{\pi k r}{m}+R_{m} \cos \frac{\pi k}{m}\right) .
$$


Here $\mathrm{PSD}_{k}$ corresponds to the power spectral density for the frequency $f_{k}=\frac{k}{2 m \Delta t}[\mathrm{~Hz}]$. The series $R_{r}$ and $\left\{B_{y_{i}}\right\}$ are given with a time interval of $\Delta t=1$ day. The whole frequency range $\left(0, \frac{1}{2 \Delta t}\right)$ is divided into $m$ sub-intervals of length $\frac{1}{2 m \Delta t}[\mathrm{~Hz}]$. Therefore, the frequency data points are $0, \frac{1}{2 m \Delta t}, \frac{2}{2 m \Delta t}, \ldots, \frac{m}{2 m \Delta t}$. We approximated the dependence of $\mathrm{PSD}_{k}$ on the frequency $f_{k}$ by a power-law function as $\mathrm{PSD}=P f^{-v}$. The values of $P$ and $v$ are derived by using the least-squares method.

\section{References}

Alania, M.V.: 2002, Acta Phys. Pol. B 33, 1149.

Alania, M.V., Iskra, K.: 1995, Adv. Space Res. 16, 241.

Alania, M.V., Iskra, K., Siluszyk, M.: 2003, Adv. Space Res. 32, 651.

Alania, M.V., Iskra, K., Siluszyk, M.: 2008a, Adv. Space Res. 41, 267.

Alania, M.V., Iskra, K., Siluszyk, M.: 2008b, Acta Phys. Pol. B 39, 2961.

Alania, M.V., Iskra, K., Siluszyk, M.: 2009 In: Proc. 21st Eur. Cosmic Ray Symp., 306.

Alania, M.V., Iskra, K., Siluszyk, M.: 2010, Adv. Space Res. 45, 1203.

Bieber, J.W., Mathaeus, W.H., Smith, C.W.: 1994, Astrophys. J. 420, 294.

Dorman, L.I.: 1975, Variations of Galactic Cosmic Rays, Moscow State University Press, Moscow, 214.

Dorman, L.I.: 2001, Adv. Space Res. 27, 601.

Gushchina, R.T., Belov, A.V., Obridko, V.N., Shelting, B.D.: 2008 In: Proc. 21st Eur. Cosmic Ray Symp., 123.

Jokipii, J.R.: 1971, Rev. Geophys. Space Phys. 9, 27.

Jokipii, J.R., Coleman, P.J.: 1968, J. Geophys. Res. 73, 5495.

Lyons, R.G.: 1996, Understanding Digital Signal Processing, Addison Wesley Longman, Boston, 544.

Shalchi, A.: 2009, Nonlinear Cosmic Ray Diffusion Theories, Springer, Berlin, 180.

Siluszyk, M., Wawrzynczak, A., Alania, M.V.: 2011, J. Atmos. Solar-Terr. Phys. 73, 1923.

Siluszyk, M., Iskra, K., Modzelewska, R., Alania, M.V.: 2005, Adv. Space Res. 35, 677.

Strauss, R.D., Potgieter, M.S.: 2014, Adv. Space Res. 53, 1015.

Yasue, S., Mori, S., Sakakibara, S., Nagashima, K.: 1982, Coupling Coefficients of Cosmic Ray Daily Variations for Neutron Monitor Stations. Rep. Cosmic Ray Res. Lab. 7, Nagoya University. 